\title{
¿HAN CAMBIADO LOS PACIENTES OFTALMOLÓGICOS QUE ACUDEN A URGENCIAS? ESTUDIO EVOLUTIVO ENTRE LOS AÑOS 1997 Y 2005
}

\section{HAVE PATIENTS WHO GO TO THE EMERGENCY DEPARTMENT CHANGED? EVOLUTIVE STUDY FOR THE YEARS 1997 AND 2005}

\author{
LEAL MA $^{1}$, BENEYTO P ${ }^{2}$, IBÁÑEZ MA ${ }^{1}$, GARCÍA A $^{1}$, FERNÁNDEZ MJ $^{1}$
}

\section{RESUMEN}

Objetivo: Estudiar el cambio en el número y perfil de los pacientes que acuden a las urgencias oftalmológicas de un hospital de segundo nivel durante los ocho últimos años.

Método: Se estudian dos grupos de pacientes que acudieron a urgencias en los mismos días, escogidos al azar, en los años 1997 y 2005. Se utilizan distintas variables: número de visitas por día, sexo, edad, franja horaria, número de exploraciones realizadas, diagnósticos y tipo de tratamiento prescrito.La comparación de proporciones se realiza mediante el estudio del intervalo de confianza y el test Chi Cuadrado.

Resultados: Son resultados estadísticamente significativos los siguientes: Aumento del número de pacientes vistos en urgencias $(\mathrm{p}<0,05)$. Aumento del diagnóstico de cuerpo extraño corneal (25\%); disminución del diagnóstico de conjuntivitis (12\%). Aumento de los casos en los que se realizan una sola exploración (19\%); aumento de la frecuencia de visitas en la franja horaria entre 18:00-21:00; disminución entre 9:00-12:00; menor frecuencia del sexo femenino (13\%) y aumento del masculino (13\%); aumento de los pacientes entre 31 y 40 años

\begin{abstract}
Objective: To study the change in number and profile of patients who came to the Emergency Department of a level 2 hospital with ophthalmic emergencies in the years 1997 and 2005.

Method: Two groups of randomly selected patients who came to the Emergency Service on the same day in the years 1997 and 2005 were studied. The study focused on the following variables: number of visits per day, gender, age, time period, number of examinations done, diagnoses and type of treatment prescribed. Comparison of proportions was carried out with a confidence interval study and Chi Square Test.
\end{abstract}

Results: The statistically significant changes $(p<0.05)$ were as follows: increase in number of patients seen; increase in the diagnosis of corneal foreign body (25\%); decrease in the diagnosis of conjunctivitis $(12 \%)$; increase in cases in which only one examination was done (19\%); increase in frequency of visits between 6 p.m. and 9 p.m. and decrease between 9 a.m. and 12 p.m; reduced attendance of those of female gender (13\%) and increase in the male gender (13\%); increase in patients between the age of 31 and 40 years $(9 \%)$; decrease

\footnotetext{
Recibido: 1/6/06. Aceptado: 13/2/07.

Servicio de Oftalmología del Complejo Hospitalario de Toledo. Toledo. España.

1 Licenciado en Medicina.

2 Doctor en Medicina.

Correspondencia:

MA Leal González

C/. Ordóñez, $27,1^{\circ} \mathrm{C}$

28911 Leganés (Madrid)

España

E-mail: m_angeleslg@yahoo.es
} 
$(9 \%)$; disminución del uso de tratamientos como la oclusión y la pomada epitelizante (7\% y $6 \%$ respectivamente); aumento del uso de analgésicos (5\%), lágrimas artificiales (5\%) y $\operatorname{AINES~(8\% );~}$ aumento de las remisiones de pacientes a su médico de atención primaria (17\%) y disminución de las revisiones oftalmológicas $(34 \%)$.

Conclusiones: El número de urgencias oftalmológicas ha aumentado en nuestra muestra en los últimos ocho años, sobre todo a expensas de pacientes varones jóvenes, con patología de origen laboral, que acuden por la tarde. Han aumentado tratamientos como las lágrimas artificiales y son remitidos a su médico de atención primaria con más frecuencia.

Palabras claves: Servicio de urgencias, lesiones del ojo, asistencia oftalmológica, epidemiología, oftalmología. in the use of treatments such as occlusion and epithelializing ointment (7\% and $6 \%$ respectively); increase in use of analgesics (5\%), artificial tears $(5 \%)$ and NSAIDs $(8 \%)$; increase in referral of patients to their primary care physician $(17 \%)$ and decrease in ophthalmology check ups (34\%).

Conclusions: The number of ophthalmological emergencies has increased in the last eight years, especially in young male patients, with work-related disease, who came in the evening. Treatments such as the use of artificial tears have increased, and patients are sent to the primary care physician more often (Arch Soc Esp Oftalmol 2007; 82: 159-166).

Key words: Emergency service, eye lesions, ophthalmic assistants, epidemiology, ophthalmology.

\section{INTRODUCCIÓN}

Los procesos agudos oftalmológicos tienen una gravedad desigual y son manejados por el propio paciente de manera muy diferente. Algunos utilizan la automedicación o la simple solicitud de fármacos en la farmacia, otros demandan consulta de medicina general u oftalmología y otros utilizan los servicios de urgencia hospitalaria (1). El carácter libre de la utilización de estos últimos los hace particularmente sensibles a los cambios de la estructura del modelo sanitario (2) (trámites burocráticos, listas de espera), a cambios de tipo social (horarios laborales o familiares) o incluso psicológicos (expectativas de rapidez y nivel asistencial). Entre los profesionales sanitarios existe la percepción creciente de que la utilización de las urgencias hospitalarias oftalmológicas está cambiando de manera significativa en los últimos tiempos, tanto en el número como en las características de las patologías tratadas.

Nuestro centro es de primer nivel que proporciona cobertura a una población de 395.514 habitantes en horario de 24 horas. Los pacientes acuden al Servicio de Urgencias bien por ser remitidos por un médico general o por un oftalmólogo o bien por su propia voluntad. Allí son examinados por un oftalmólogo o residente de oftalmología bajo su supervisión, son tratados si es necesario y derivados en ocasiones para revisión.
Nuestro objetivo es estudiar el cambio en el número y perfil de los pacientes que acuden a las urgencias oftalmológicas de un hospital de primer nivel durante los ocho últimos años. Para ello estudiaremos dos grupos de pacientes que acudieron en los mismos días escogidos de manera aleatoria de los años 1997 y 2005 a los servicios de urgencias de oftalmología de nuestro centro. De todos ellos se consignan variables demográficas (edad, sexo), procedencia (viene libremente o derivado por un médico u oftalmólogo), franja horaria en la que acuden a la urgencia, número de exploraciones realizadas, diagnósticos encontrados, tratamiento y número de derivación a otras consultas.

\section{SUJETOS, MATERIAL Y MÉTODOS}

\section{Aleatorización de los pacientes}

Se recogieron consecutivamente las hojas de exploración de todos los pacientes que acudieron a urgencias de oftalmología en cinco días escogidos al azar del año 1997 y los 5 días correspondientes de 2005 (se considera correspondiente el mismo día de la semana de la misma semana del mismo mes, evitando aquellos que tuvieran un día festivo en esa misma semana o en las semanas adyacentes a fin de evitar sesgos). 


\section{Variables estudiadas:}

De las hojas de exploración se recogieron las siguientes variables:

- Número de visitas por día y porcentaje respecto al total de pacientes del servicio de urgencias.

- Sexo.

- Rango de edad establecido en 0-10, 11-20, $21-30,31-40,41-50,51-60,61-70,71-80,>81$ años.

- Franja horaria establecido en 9-12 h, 12-15 h, 15-18 h, 18-21 h, 21-9 h.

- Número de exploraciones realizadas (se consideran como tales la toma de la agudeza visual, biomicroscopía, motilidad ocular externa, estudio de reflejos pupilares, presión intraocular, fondo de ojo y otros).

- Motivos de consulta (ojo rojo, sensación de cuerpo extraño, dolor, molestias, disminución de agudeza visual y otros).

- Diagnóstico, cuanto está explícitamente consignado (cuerpo extraño corneal, conjuntivitis, problemas corneales y otros).

- Tratamiento prescrito, agrupado por grupos terapéuticos de fármacos: antibióticos, corticoides, lágrimas artificiales, ciclopléjico, oclusión, pomada epitelizante, analgésicos, suero fisiológicos, AINES y otros. Cada enfermo puede haber recibido fármacos de varios grupos o ninguno.

\section{Estudio estadístico}

Se utiliza la diferencia de proporciones entre las muestras, estudiando el intervalo de confianza pata un nivel de significación del 90\%. Se considera estadísticamente significativo si el 0 no está incluido en el intervalo de confianza. Se utiliza el test de Chi cuadrado para comparar el número de pacientes totales.

\section{RESULTADOS}

Se estudiaron 194 formularios de urgencia oftalmológica, 78 provenientes de pacientes de 1997 y 116 de 2005. Estas cifras representan el 7\% del total de urgencias hospitalarias de los mismos días en 1997 y el 5,9\% en 2005. Los diagnósticos consignados se muestran en la tabla I y figura 1 . El motivo de consulta y el número de exploraciones realizadas se pueden ver en las tabla II, figura 2 y tabla III, figura 3 respectivamente. La distribución de las urgencias en los diferentes tramos horarios se muestran en la tabla IV y figura 4. La distribución en cuanto a sexo y edad se muestran en las tablas $\mathrm{V}$ y VI, y en las figuras 5 y 6 respectivamente. La tabla VII y figura 7 muestra los tratamientos prescritos y la tabla VIII y figura 8 la distribución de pacientes mandados a revisión. Las tablas IX y X y las figuras 9 y 10 representan el número de pacien-

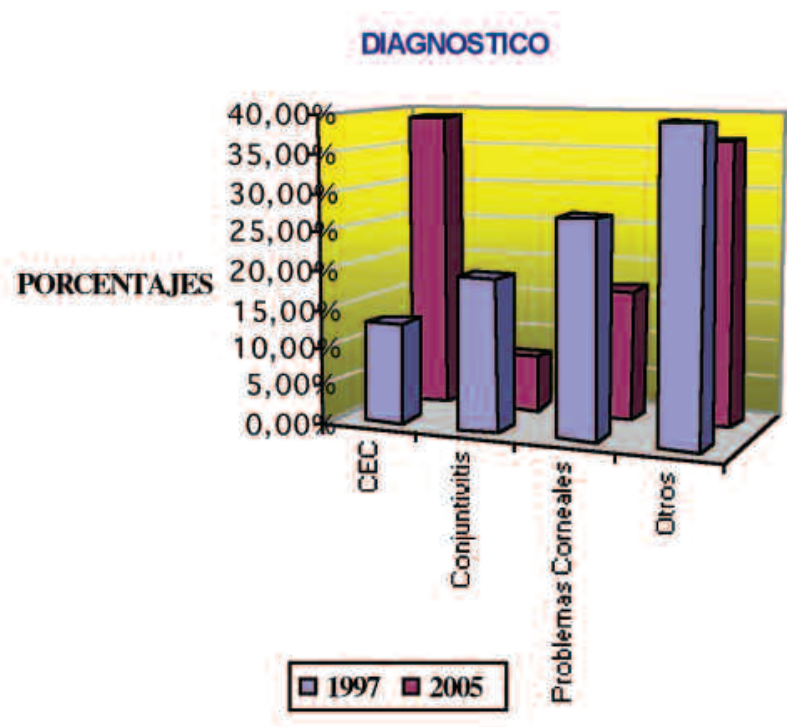

Fig. 1: Diagnósticos consignados en 1997 y 2005 (CEC: cuerpo extraño corneal).

Tabla I. Diagnósticos consignados

\begin{tabular}{|c|c|c|c|c|c|c|c|c|c|}
\hline \multicolumn{4}{|c|}{1997} & \multicolumn{4}{|c|}{2005} & Dif \% & IC \\
\hline \multirow{4}{*}{$\begin{array}{l}\text { Diagnósticos } \\
\text { consignados } \\
61(78,2 \%)\end{array}$} & CEC & 8 & 13,10 & \multirow{4}{*}{$\begin{array}{l}\text { Diagnósticos } \\
\text { consignados } \\
41(35,3 \%)\end{array}$} & CEC & 16 & $39 \%$ & $25 \%$ & $0,11,0,39 \mathrm{ES}$ \\
\hline & Conjuntivitis & 12 & $19,60 \%$ & & Conjuntivitis & 3 & $7,30 \%$ & $-0,12$ & $-0,004,-0,22 \mathrm{ES}$ \\
\hline & $\begin{array}{l}\text { Problemas } \\
\text { corneales }\end{array}$ & 17 & $27,80 \%$ & & $\begin{array}{l}\text { Problemas } \\
\text { corneales }\end{array}$ & 7 & $17 \%$ & $-0,1$ & $0,03,-0,23$ \\
\hline & Otros & 24 & $39,50 \%$ & & Otros & 15 & $36,70 \%$ & $-0,02$ & $0,13,-0,18$ \\
\hline
\end{tabular}

CEC: cuerpo extraño corneal; ES: estadísticamente significativo. 
Tabla II. Número de exploraciones realizadas

\begin{tabular}{lrrrrrr}
\hline N. ${ }^{\circ}$ exploraciones & \multicolumn{2}{c}{1997} & \multicolumn{2}{c}{2005} & Dif $\%$ & IC \\
\hline 1 & 33 & $55,90 \%$ & 71 & $75,50 \%$ & 0,19 & $0,06,0,32$ ES \\
2 & 11 & $18,60 \%$ & 8 & 8,50 & $-0,033$ & $-0,09,0,02$ \\
3 & 6 & $10,10 \%$ & 6 & $6,30 \%$ & $-0,037$ & $-0,12,0,35$ \\
4 & 6 & $10,10 \%$ & 5 & $5,30 \%$ & $-0,48$ & $-0,13,0,22$ \\
5 & 1 & $1,60 \%$ & 4 & $4,20 \%$ & 0,025 & $-0,03,0,07$ \\
6 & 2 & $3,30 \%$ & 0 & $0 \%$ & $-0,033$ & $-0,09-0,002$ \\
\hline \hline
\end{tabular}

ES: estadísticamente significativo.

Tabla III. Motivos de consulta

\begin{tabular}{lcccccc}
\hline Síntomas & \multicolumn{2}{c}{1997} & \multicolumn{2}{c}{2005} & Dif $\%$ & IC \\
\hline Ojo rojo & 14 & $18,10 \%$ & 24 & $19,20 \%$ & 0,01 & $-0,087,0,098$ \\
SCE & 12 & $15,50 \%$ & 21 & $16,80 \%$ & 0,012 & $-0,08,0,095$ \\
Dolor & 6 & $7,70 \%$ & 15 & $12 \%$ & 0,04 & $-0,03,0,1$ \\
Molestias & 7 & $9 \%$ & 18 & $14,40 \%$ & 0,05 & $-0,02,0,12$ \\
$\downarrow$ AV & 6 & $7,70 \%$ & 8 & $6,40 \%$ & $-0,01$ & $-0,08,0,04$ \\
Otros & 32 & $42 \%$ & 39 & $31,20 \%$ & $-0,1$ & $-0,21,0,009$ \\
\hline \hline
\end{tabular}

SCE: sensación de cuerpo extraño.

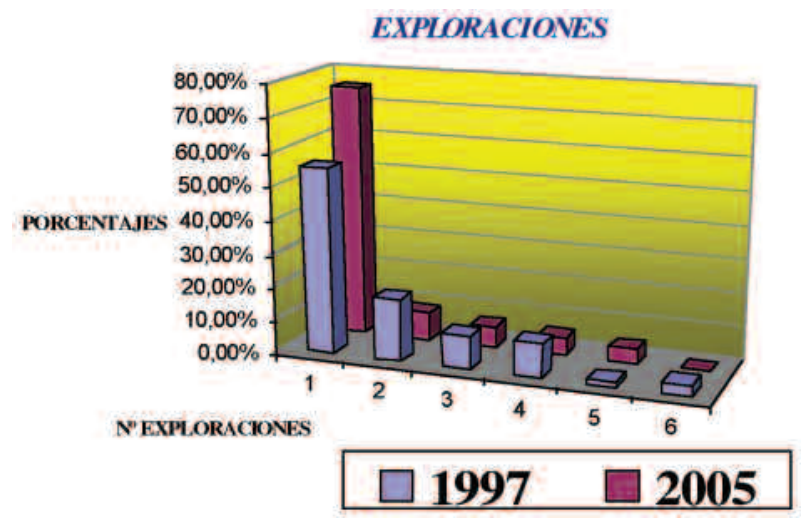

Fig. 2: Número de exploraciones realizadas en 1997 y 2005.

tes que acuden a las urgencias de oftalmología por día y por año.

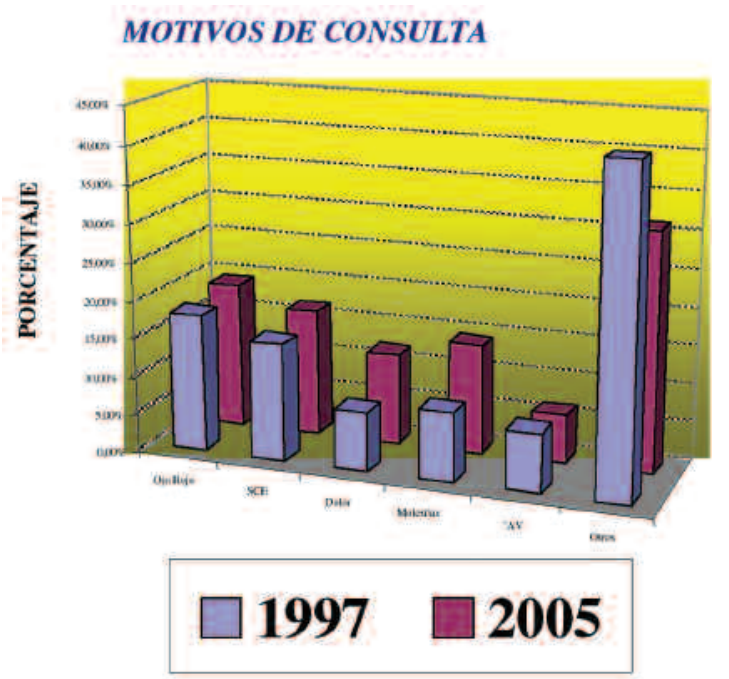

Fig. 3: Motivos de consulta realizados en 1997 y 2005 (SCE: sensación cuerpo extraño).

Tabla IV. Distribución de las urgencias según las franjas horarias

\begin{tabular}{lcccccc}
\hline Horas & \multicolumn{2}{c}{1997} & & 2005 & Dif $\%$ & IC \\
\hline 9:00-12:00 & 24 & $34,20 \%$ & 21 & $18,90 \%$ & $-0,17$ & $-0,28,-0,06$ ES \\
$12: 00-15: 00$ & 10 & $14,20 \%$ & 20 & $18 \%$ & 0,02 & $-0,07,0,11$ \\
$15: 00-18: 00$ & 14 & $20 \%$ & 28 & $25,20 \%$ & 0,04 & 0,1 \\
$18: 00-21: 00$ & 9 & $12,80 \%$ & 27 & $24,30 \%$ & $-0,07,0,14$ \\
$21: 00-9: 00$ & 13 & $18,50 \%$ & 15 & $13,50 \%$ & $-0,02$ & $-0,16,0,03$ \\
\hline \hline
\end{tabular}

ES: estadísticamente significativo. 


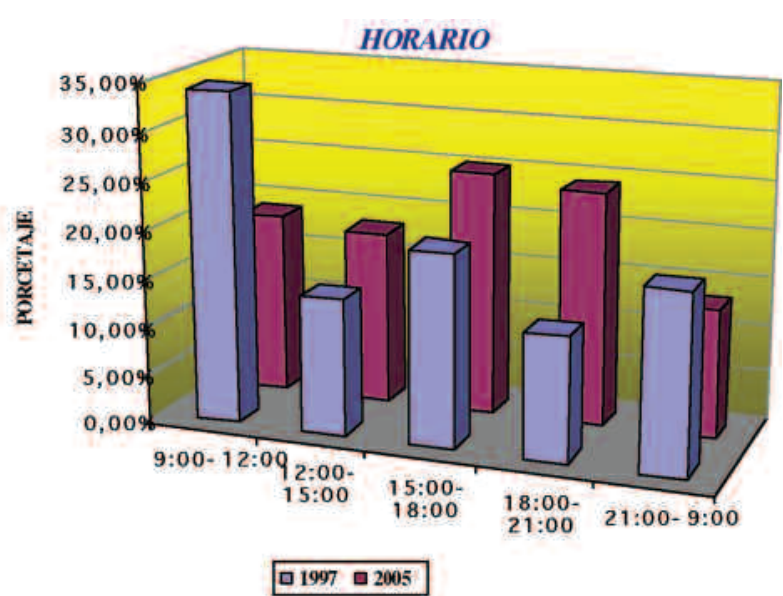

Fig. 4: Distribución de urgencias hospitalarias, según la franja horaria, en 1997 y 2005.

Tabla V. Distribución de las urgencias según el sexo

\begin{tabular}{lcccrrr}
\hline Sexo & 1997 & 2005 & Dif $\%$ & IC \\
\hline Hombre & 38 & $54,20 \%$ & 75 & $67,50 \%$ & 0,13 & $0,01,0,25 \mathrm{ES}$ \\
Mujer & 32 & $45,70 \%$ & 36 & $32,50 \%$ & $-0,13$ & $-0,01,0,13 \mathrm{ES}$ \\
\hline \hline
\end{tabular}

ES: estadísticamente significativo.

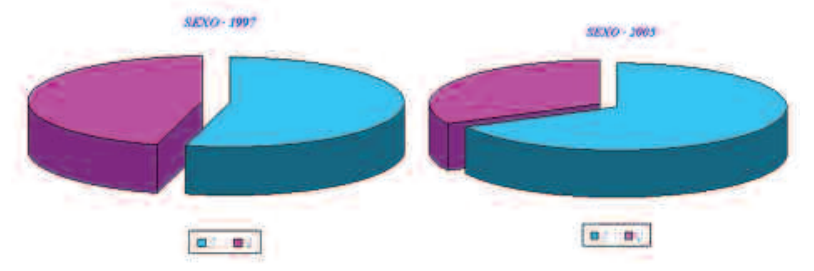

Fig. 5: Distribución de urgencias, según el sexo, en 1997 y 2005.
Tabla VI. Distribución de las urgencias según la edad

\begin{tabular}{lrrrlrl}
\hline Años & & 1997 & & 2005 & Dif $\%$ & IC \\
\hline $0-10$ & 6 & $8,60 \%$ & 11 & $10 \%$ & 0,01 & $-0,06,0,08$ \\
$11-20$ & 4 & $5,70 \%$ & 3 & $2,70 \%$ & $-0,03$ & $-0,09,0,01$ \\
$21-30$ & 13 & $18,80 \%$ & 12 & $11 \%$ & $-0,07$ & $-0,17,0,008$ \\
$31-40$ & 7 & $10,10 \%$ & 22 & $20,10 \%$ & 0,09 & $0,005,0,18 \mathrm{ES}$ \\
$41-50$ & 6 & $8,60 \%$ & 13 & $11,90 \%$ & 0,03 & $-0,05,0,10$ \\
$51-60$ & 13 & $18,80 \%$ & 13 & $11,90 \%$ & $-0,07$ & $-0,16,0,01$ \\
$61-70$ & 10 & $14,40 \%$ & 18 & $16,50 \%$ & 0,01 & $-0,07,0,10$ \\
$71-80$ & 9 & $13 \%$ & 13 & $11,90 \%$ & $-0,01$ & $-0,10,0,05$ \\
$>81$ & 1 & $1,40 \%$ & 4 & $3,60 \%$ & 0,02 & $-0,03,0,06$ \\
\hline \hline
\end{tabular}

ES: estadísticamente significativo.

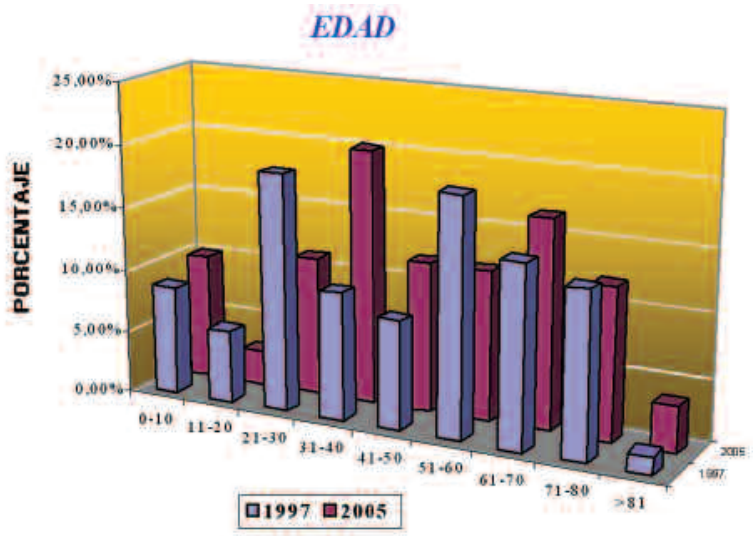

Fig. 6: Distribución de urgencias, según la edad, en 1997 y 2005.

\section{DISCUSIÓN}

Existen pocos estudios que estudien la asistencia oftalmológica urgente de todas las patologías, siendo más frecuente el estudio de la casuística de las patologías más graves $(3,4)$, generalmente las de origen traumático (5-9). El porcentaje de urgencias

Tabla VII. Tratamientos prescritos

\begin{tabular}{lcccccc}
\hline Tipo & \multicolumn{2}{c}{1997} & \multicolumn{2}{c}{2005} & Dif $\%$ & IC \\
\hline Antibióticos & 32 & $22,30 \%$ & 57 & $25,20 \%$ & 0,02 & $-0,04,0,09$ \\
Ciclopéjico & 24 & $16,70 \%$ & 25 & $11 \%$ & $-0,05$ & $-0,12,0,002$ \\
Oclusión & 22 & $15,30 \%$ & 17 & $7,60 \%$ & $-0,07$ & $-0,13,-0,02$ ES \\
Corticoide & 15 & $10,40 \%$ & 19 & $8,40 \%$ & $-0,02$ & $-0,07,0,02$ \\
Pomada epitelizante & 11 & $7,60 \%$ & 2 & $0,80 \%$ & $-0,06$ & $-0,11,0,03$ ES \\
Analgésico & 3 & $2 \%$ & 18 & $7,90 \%$ & $-0,05$ & $0,01,0,09$ ES \\
Lágrimas artificiales & 2 & $1,30 \%$ & 16 & $7 \%$ & 0,05 & $0,02,0,09$ ES \\
S. Fisiológico & 2 & $1,30 \%$ & 10 & $4,40 \%$ & 0,03 & $-0,002,0,05$ \\
AINES & 0 & $0 \%$ & 19 & $8,40 \%$ & 0,08 & $0,05,0,11$ ES \\
Otros & 33 & $23,10 \%$ & 45 & $19,30 \%$ & $-0,03$ & $-0,10,0,03$ \\
\hline \hline
\end{tabular}

ES: estadísticamente significativo. 


\section{TRATAMIENTOS}

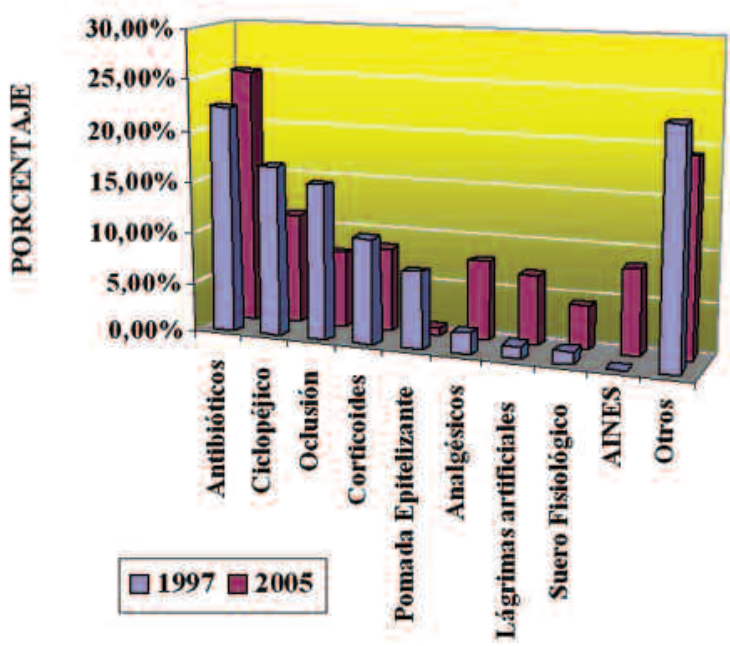

Fig. 7: Tratamientos prescritos en 1997 y 2005.

Tabla VIII. Distribución de las revisiones

\begin{tabular}{|c|c|c|c|c|c|}
\hline & \multicolumn{2}{|c|}{1997} & 2005 & Dif $\%$ & IC \\
\hline $\begin{array}{l}\text { Revisión } \\
\text { oftalmol. }\end{array}$ & & $78 \%$ & $3151,60 \%$ & $-0,34$ & $-0,47,-0,16 \mathrm{ES}$ \\
\hline 1. ${ }^{\mathrm{a}}$ consulta & & $10,70 \%$ & $711,60 \%$ & 0,007 & $-0,14,0,09$ \\
\hline MAP & 1 & $3,50 \%$ & $1525 \%$ & 0,17 & $0,04,0,27 \mathrm{ES}$ \\
\hline Mutua & 2 & $7,10 \%$ & $610 \%$ & 0,01 & $-0,11,0,10$ \\
\hline MI & 0 & $0 \%$ & $1 \quad 1,60 \%$ & 0,01 & $-0,07,0,06$ \\
\hline
\end{tabular}

MAP: médico atención primaria; MI: medicina interna; ES: estadísticamente significativo.

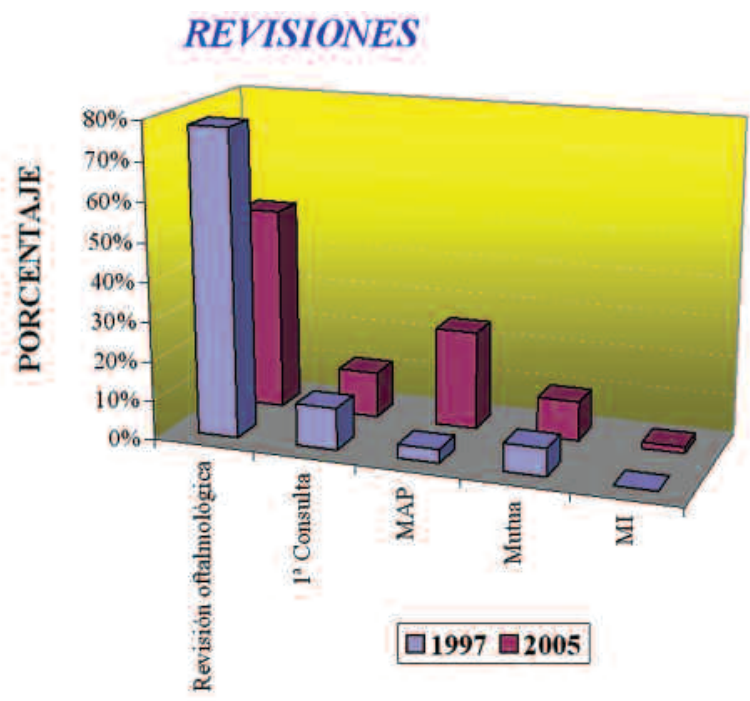

Fig. 8: Distribución de las revisiones en 1997 y 2005.
Tabla IX. Media del número de pacientes que acuden a urgencias oftalmológicas por día

\begin{tabular}{|c|c|c|c|c|}
\hline & $\begin{array}{c}1997 \\
\text { Media/día }\end{array}$ & $\begin{array}{c}2005 \\
\text { Media/día }\end{array}$ & Dif \% & IC \\
\hline Hospital & 222,6 & 388 & & \\
\hline Oftalmología & $15,67 \%$ & $23,2 \quad 5,90 \%$ & $-0,008$ & $-0,04,0,02$ \\
\hline
\end{tabular}

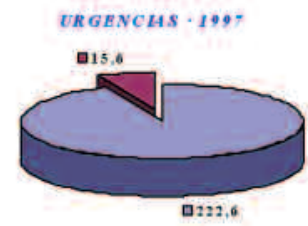

\section{$\square$ Hospital Oftalmología}

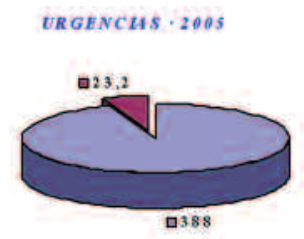

\section{$\square$ Hospital $\square$ Oftalmología}

Fig. 9: Media del número de pacientes que acuden a urgencias oftalmológicas por día, en 1997 y 2005.

oftalmológicas sobre el total de urgencias obtenido en nuestra muestra (5,9\% en 2005 y $7 \%$ en 1997) es similar a la de otras series [5,6\% Sánchez Tocino (10); Girard 5\% (11); Nash 7,6\% (12); 7\% Tan (13)]. El porcentaje de hombres es mayor (54\% en 1997 y $64 \%$ en 2005), lo que también fue observado por Kumar (14) y Nash. Sánchez Tocino encuentra una mayor prevalencia de mujeres $(53,2 \%)$, lo que puede ser debido a que en su sistema de urgencias no existe presencia física del oftalmólogo en el intervalo de la tarde, que por razones laborales suele ser el más frecuentado por varones.

Existen unas variables en las que la diferencia entre los años 1997 y 2005 es claramente significativa. Estas incluyen el número de visitas por día (de 14 a 23 pacientes de media), el sexo masculino (de 54 a $67 \%$ ) (15-19), la edad comprendida entre 3140 años (de 8 a 18\% del total), los síntomas catalogados como «molestias»o «dolor» (16 a 26\%) y el porcentaje de pacientes que acuden en el rango horario que va desde las 18 a 21 horas (12 al 24\%).

Tabla X. Número de pacientes totales que acuden a urgencias oftalmológicas por año

\begin{tabular}{lcc}
\hline 1997 & 2005 & $\mathrm{Chi}^{2}$ \\
\hline 66 & 111 & $4,117 \mathrm{p} \leq 0,05$ ES \\
\hline \hline
\end{tabular}


Igualmente han aumentado el número de pacientes remitidos por el médico de cabecera o por el oftalmólogo (del 1,2 al 12\%).

Con respecto a las razones de estos cambios, nuestra impresión solo puede ser especulativa. El aumento del número de pacientes y de las patologías banales a las que se ha prescrito solo un tratamiento sintomático puede ser un síntoma de evitación de conductas generales programadas, que presuponen una mayor molestia burocrática y suelen estar sujetas a listas de espera. Un horario laboral exigente puede ser la causa del desplazamiento horario de las urgencias hacia la última hora de la tarde, así como un mayor uso de las mismas por los varones, que en nuestro área presentan una mayor actividad laboral que las mujeres. La conducta ofrecida por el oftalmólogo también es significativa: se ofrece un menor número de exploraciones clínicas, pero se remite a revisión en un número mayor de ocasiones. Lo primero puede deberse a la menor gravedad de los motivos de consulta, mientras que lo segundo tal vez sea debido a un aumento significativo de la presión legal.

\section{BIBLIOGRAFÍA}

1. Mulla ZD, Margo CE. Hospitalitation for nontraumatic disorders of the eye and ocular adnexa: analysis of the Florida agency for health care administration data set. Arch Ophthalmol 2004; 122: 262-266.

2. Steinbrook $R$. The role of the emergency department. $N$ Engl J Med 1996; 334: 657-658.

3. Edwards RS. Ophthalmic emergencies in a district general hospital casualty department. Br J Ophthalmol 1987; 71: $938-942$.

4. Tabandeh H, Kon C, Rostron C, Luke I. Systemic conditions in the Emergency Eye Clinic. Eur J Ophthalmol 1997; 7: 387-390.

5. Schein OD, Hibberd PL, Shingleton BJ, Kunzweiler T, Frambach DA, Seddon JM, et al. The spectrum and burden of ocular injury. Ophthalmology 1988; 95: 300-305.
6. Desai P, MacEwen CJ, Baines P, Minassian DC. Incidence of cases of ocular trauma admitted to hospital and incidence of blinding outcome. Br J Ophthalmol 1996; 80: 592-596.

7. Pieramici DJ, Sternberg P Jr, Aaberg TM Sr, Bridges WZ $J r$, Capone A Jr, Cardillo JA, et al. A system for classifying mechanical injuries of the eye (globe). The Ocular Trauma Classification Group. Am J Ophthalmol 1997; 123: 820-831.

8. Andreotti $G$, Lange JL, Brundage Jr. The nature, inciden$c e$, and impact of eye injuries among US military personnel: implications for prevention. Arch Ophthalmol 2001; 119: 1693-1697.

9. Kuhn F, Morris R, Witherspoon CD, Heimann K, Jeffers $J B$, Treister G. A standardized classification of ocular trauma. Ophthalmology 1996; 103: 240-243.

10. Sánchez Tocino H, Galindo Ferreiro A, Iglesias Cortinas D, Galindo Alonso J, Fernández Muñoz M. Estudio epidemiológico de las urgencias oftalmológicas en un hospital general. Arch Soc Esp Oftalmol 2004; 79: 425-431.

11. Girard B, Bourcier F, Agdabede I, Laroche L. Activity and epidemiology in an ophthalmological emergency center. $J$ Fr Ophtalmol 2002; 25: 701-711.

12. Nash EA, Margo CE. Patterns of emergency department visits for disorders of the eye and ocular adnexa. Arch Ophthalmol 1998; 116: 1222-1226.

13. Tan MM, Driscoll PA, Marsden JE. Management of eye emergencies in the accident and emergency department by senior house officers: a national survey. J Accid Emerg Med 1997; 14: 157-158.

14. Kumar NL, Black D, McClellan K. Daytime presentations to a metropolitan ophthalmic emergency department. Clin Experiment Ophthalmol 2005; 33: 586-592.

15. Jones NP, Hayward JM, Khaw PT, Claoue CM, Elkington AR. Function of an ophthalmic "accident and emergency» department: results of a six month survey. Br Med J (Clin Res Ed) 1986; 292: 188-190.

16. Bhopal RS, Parkin DW, Gillie RF, Han KH. Pattern of ophthalmological accidents and emergencies presenting to hospitals. J Epidemiol Community Heatlh 1993; 47: 382-387.

17. Karlson TA, Klein BE. The incidence of acute hospital-treated eye injures. Arch Ophthalmol 1986; 104: 1473-1476.

18. McGwin G Jr, Xie A, Owsly C. Rate of eye injury in the United States. Arch Ophthalmol 2005; 123: 970-976.

19. McGwin G Jr, Owsley C. Incidence of emergency department-treated eye injury in the United States. Arch Ophthalmol 2005; 123: 662-666. 